\title{
Electro-Acupuncture Stimulation Effects on Duodenal Motility in Anesthetized Rats
}

\author{
Eitaro NOGUCHI, Hideo OHSAWA*, Hideki TANAKA*, \\ Hiroko IKEDA ${ }^{\dagger}$, and Yoshihiro AIKAWA \\ Laboratory of Acupuncture and *Laboratory of Physiology, Tsukuba College of Technology, \\ Tsukuba, 305-0821 Japan; and ${ }^{\dagger}$ Graduate School of Humanities and Sciences, \\ Ochanomizu University, Bunkyo-ku, Tokyo, 112-0012 Japan
}

\begin{abstract}
The effect of electro-acupuncture stimulation (EAS) on duodenal motility was examined in anesthetized, artificially ventilated rats. EAS was applied to the abdominal area or to a hindpaw for $30 \mathrm{~s}$ at stimulus intensities of $0.1-$ $10.0 \mathrm{~mA}$ with a stimulus frequency of $20 \mathrm{~Hz}$. The duodenal motility was measured using the balloon method at a position about $1.5 \mathrm{~cm}$ caudal from the pylorus. Duodenal motility was inhibited by EAS at intensities of more than $5.0 \mathrm{~mA}$ (suprathreshold of group IV afferent excitation) when applied to the abdominal area. The duodenal inhibitory response existed after bilateral vagotomy or spinal transection, but was abolished by sectioning bilateral splanchnic nerves. Duodenal motility was facilitated by EAS at intensities of more than $2.0 \mathrm{~mA}$ (subthreshold of group
\end{abstract}

IV, and supurathreshold for groups II+III afferent excitation) when applied to a hindpaw. The duodenal facilitatory response by EAS to a hindpaw existed after sectioning the splanchnic nerves, but disappeared after bilateral vagotomy or spinal transection. Furthermore, repetitive electrical stimulation of vagal efferent nerves enhanced duodenal motility, while repetitive electrical stimulation of the splanchnic efferent nerves inhibited the motility. It was concluded that the inhibitory response of duodenal motility elicited by EAS to the abdominal area is a spinal reflex response involving splanchnic inhibitory efferent nerves, and the enhanced response of duodenal motility by EAS to a hindpaw is a supraspinal reflex response involving vagal excitatory nerves. [Japanese Journal of Physiology, 53, 1-7, 2003]

Key words: duodenal motility, vagal nerve, splanchnic nerve, electro-acupuncture, rat.

A Acupuncture has been used for over 1,000 years in Asia for the treatment of various diseases including gastrointestinal dysfunction (see reviews by O'Connor and Bensky [1] and Li [2]). The mechanisms underlying the effects of acupuncture on gastrointestinal function have long awaited clarification.

Sato et al. analyzed the reflex properties of gastric motility elicited by cutaneous sensory stimulation using anesthetized rats, including the central nervous system and the efferent autonomic nerves involved [3]. In their preparation, emotional factors due to somatic sensory stimulation were eliminated using anesthetized animals. They found that gastric motility, inhibited by pinching the abdominal skin through exciting splanchnic gastric efferent nerves, is a pro- priospial reflex, while motility facilitated by pinching a hindpaw through exciting vagal gastric efferent nerves is a supraspinal reflex $[4,5]$.

It was found, using anesthetized rats, that acupuncture-like stimulation by manually moving a needle inserted into the skin and its underlying muscles excited somatic afferent nerves and produced both inhibitory and excitatory reflex responses of gastric motility similar to those elicited by cutaneous pinching. The contributions of splanchnic and vagal gastric efferent nerves and involvement of the spinal and supraspinal central structures were similar to those involved in the responses by pinching the skin [6]. Noguchi and Hayashi found an increase in gastric acid secretion following electro-acupuncture of a hindlimb in anes-

Received on September 26, 2002; accepted on November 28, 2002

Correspondence should be addressed to: Eitaro Noguchi, Laboratory of Physiology, Tsukuba College of Technology, Tsukuba, 305-0821 Japan. Tel: +81-298-58-9540, Fax: +81-298-58-9549, E-mail: enoguchi@k.tsukuba-tech.ac.jp 
thetized rats [7]. This gastric acid secretion response was suggested to be a reflex using somatic nerves as afferents and gastric vagus as efferents.

The effect of somatic sensory stimulation on duodenal motility was investigated in anesthetized rats [8]. They stimulated the neck, chest, and abdominal skin, and found that pinching the abdominal skin produced a reflex inhibition of duodenal motility by exciting splanchnic sympathetic efferent nerves. The reflex was a spinal reflex.

In a continuation of these experimental studies, the present work aimed to examine if acupuncture stimulation affects duodenal motility in anesthetized animals whose emotional factor has been suppressed. In the present study, we chose two areas for acupuncture stimulation (i.e., the abdominal area and a hindpaw). Abdominal pinching was shown to produce an inhibitory response of gastric and duodenal motility, and hindpaw stimulation was shown to produce a facilitatory response for gastric motility, although hindpaw stimulation has not been tested for duodenal motility. We used electro-acupuncture stimulation (EAS) to these two areas because we could precisely control the stimulus intensity. When we observed the response of duodenal motility following electro-acupuncture, we tried to find the contribution of afferent somatic and efferent autonomic nerves to the response and the involvement of the spinal and supraspinal central structures using a spinal transection procedure at the thoracic spinal level.

\section{MATERIALS AND METHODS}

General experimental conditions. The experiments were performed on 27 adult male Wistar rats $(230-390 \mathrm{~g})$ anesthetized with urethane $(1.3 \mathrm{~g} / \mathrm{kg}$, I.P.). The trachea was cannulated, animals were immobilized with gallamine triethiodide $(20 \mathrm{mg} / \mathrm{kg}$, I.V.), and respiration was artificially maintained using a respirator (SN-480-7, Shinano, Tokyo, Japan) to keep respiratory conditions constant. The end-tidal $\mathrm{CO}_{2}$ concentration, monitored with a gas monitor $(1 \mathrm{H} 26$, NEC San-ei, Tokyo, Japan), was kept at about 3.0\% by controlling the volume and frequency of the respirator. Systemic blood pressure was continuously recorded through a catheter in the right common carotid artery. The jugular vein was cannulated for infusion of the necessary solutions. Core body temperature, monitored in the rectum, was maintained at $37.0-38.0^{\circ} \mathrm{C}$ using a body temperature control system containing a thermostatically regulated DC current heating pad and infrared lamp (ATB-1100, Nihon Kohden, Tokyo, Japan). During the experiment, the depth of anesthesia was routinely judged by observing the animal's blood pressure and heart rate. Whenever these conditions were unstable, additional urethane (about $0.1 \mathrm{~g} / \mathrm{kg}$ ) was intravenously administered.

Recording of duodenal motility. The rats were kept in a supine position. The midline of the abdomen was cut and a small balloon about $1.0 \mathrm{~cm}$ diameter, made of condom rubber, was inserted into the duodenum through a small incised hole $(2-3 \mathrm{~mm})$ about $3 \mathrm{~cm}$ caudal from the pylorus. Special care was taken not to damage the blood vessels. The balloon was fixed around $1.5 \mathrm{~cm}$ caudal from the pylorus during the experiments. The balloon was connected, via a catheter (outer diameter: $1 \mathrm{~mm}$ ), to a pressure transducer (TP-400T, Nihon Kohden) to measure duodenal pressure continuously and to record it on a polygraph pen recorder (RM-6000, Nihon Kohden). Another catheter (inner diameter: $1 \mathrm{~mm}$ ) was also inserted into the same hole by incision in order to drain digestive juices secreted from the duodenum.

Electro-acupuncture stimulation (EAS). In 19 of the rats used, the stimuli were delivered to the abdomen (about $3-4 \mathrm{~cm}$ lateral to the midline and $3-4 \mathrm{~cm}$ caudal to the processus xiphoideus) and a hindpaw (interdigital space of the 2nd and 3rd digits). Two stainless steel acupuncture needles of $160 \mu \mathrm{m}$ diameter (Seirin, Shizuoka, Japan), were inserted obliquely into the skin and underlying tissues of the left hindpaw or abdomen about $5 \mathrm{~mm}$ apart and to a depth of about $5 \mathrm{~mm}$. EAS was performed by passing a square-wave pulse current between these two needles using a constant-current electrical stimulator (SEN-7203, Nihon Kohden). The parameters of electrical stimulation were $0.5 \mathrm{~ms}$ duration, $0.1-10.0 \mathrm{~mA}$ intensity, and $20 \mathrm{~Hz}$ frequency for $30 \mathrm{~s}$. As the maximal responses of blood flow and pupillary dilation to electrical stimulation appeared at $20 \mathrm{~Hz}$ in our previous studies $[9,10]$, we used $20 \mathrm{~Hz}$ stimulation in the present experiments.

Each train of these stimuli was delivered to the animal after observing the stability and recovery of duodenal motility after the preceding stimulus, and it was usually necessary to wait for more than 5 min between each stimulus train.

Surgical severance of the spinal, sciatic, and femoral nerves. The spinal nerves between the T8 and T13 levels were separated from the surrounding tissues and cut along the spine in four cases. Femoral and sciatic nerves were cut in the femoral area in four cases.

Recording of action potentials from the somatic afferent nerves. In four rats, the left saphenous and tibial nerves were separated from the 
surrounding tissues at the femoral area and cut. In four rats, cutaneous nerve branches of the T11-12 intercostal nerves were also separated from the surrounding tissues and cut at the central position. The action potentials, elicited by a single shock to a hindpaw via the acupuncture needles at various intensities, were recorded by a pair of wire electrodes placed at the distal cut end of the nerves. The distance between the stimulation area and proximal recording electrode of a pair of electrodes at the saphenous, tibial, and T11-12 intercostal nerves was about 7.0-8.0, 4.6-6.0, and $4.9-5.2 \mathrm{~cm}$, respectively. Action potentials were amplified (using a time constant of $0.3 \mathrm{~s}$ ), averaged using an averaging computer (ATAC 3700, Nihon Kohden), and displayed on a storage oscilloscope. Conduction velocities of myelinated and unmyelinated fibers were calculated by measuring the length of a nerve between the stimulating area and proximal recording electrode, and also measuring the latencies of both evoked action potentials.

Severance of autonomic nerves innervating the duodenum. Bilateral severance of the splanchnic nerve just beneath the diaphragm was performed on three rats. Bilateral severance of the vagal nerves was performed on six rats at the cervical level during experiments.

Spinal transection. The spinal cord was transected at the 2nd thoracic (T2) level in two rats. After transection of the spinal cord, 4\% Ficoll (Ficoll 70, Pharmacia, Uppsala, Sweden) was intravenously injected to maintain the systolic blood pressure above $60 \mathrm{mmHg}$.

Stimulation of autonomic nerves innervating the duodenum. Vagi were sectioned bilaterally at the cervical level, and the effects of vagus stimulation on duodenal motility were examined in four cases. The peripheral cut end of one side was stimulated by a pair of wire electrodes. The parameters of electrical stimulation were $0.5 \mathrm{~ms}$ duration, 10 and $50 \mathrm{~Hz}$ frequency, and 1 and $5 \mathrm{~V}$ intensity for $15 \mathrm{~s}$. The left splanchnic nerve was dissected and isolated from the other tissues retroperitoneally, cut close to the diaphragm, and the peripheral cut end was electrically stimulated in four cases. Dissected nerves were kept for stimulation in a warm $\left(37^{\circ} \mathrm{C}\right)$ liquid paraffin pool.

Statistic analysis. Data are expressed as mean \pm SEM. Statistical testing was performed using the paired $t$-test.

\section{RESULTS}

\section{Duodenal motility under resting condition}

When the balloon was inserted into the duodenum and filled with about $0.2 \mathrm{ml}$ of warm saline, the baseline pressure was around $100 \mathrm{mmH}_{2} \mathrm{O}$ and there were two wave patterns: small, fast, rhythmic waves (frequency: 5-49/min; amplitude: $2-40 \mathrm{mmH}_{2} \mathrm{O}$ ); and large, slow, irregular waves (amplitude: 180-300 $\mathrm{mmH}_{2} \mathrm{O}$ ) as described by Sato and Terui [8]

\section{Effect of EAS on duodenal motility}

In the present study, we mainly examined the effect of EAS on the small, fast, rhythmic waves of duodenal motility.

\section{EAS of a hindpaw.}

Effects of EAS of a hindpaw on duodenal motility. Figure 1A shows typical responses of duodenal motility following EAS with $20 \mathrm{~Hz}$ at various stimulation intensities for $30 \mathrm{~s}$. Figure 1C summarizes the responses obtained from all 11 rats tested. When the stimulation intensity was less than $1.5 \mathrm{~mA}$, there was no significant response of duodenal motility. EAS at
Fig. 1. Effect of EAS of a hindpaw at various intensities at $20 \mathrm{~Hz}$ for $30 \mathrm{~s}$ on duodenal motility. A: Sample recordings of the responses of duodenal motility to EAS of a hindpaw. Stimulation intensities are indicated on the left side of each trace. The thin dashed lines and thick horizontal bars on the abscissa indicate the time of stimulation. B: Sample recordings of the evoked potentials of myelinated and unmyelinated fibers from the saphenous nerve by EAS of a hindpaw. C: Summarized graphs of relationships between stimulation intensity and magnitude of change in duodenal motility. Changes in mean duodenal pressure are expressed as percentages of the mean prestimulation duodenal pressure. ${ }^{* *} p<0.01$ : significantly different from prestimulation control value using a paired $t$-test. $T_{\|}$,

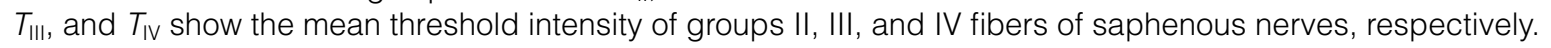
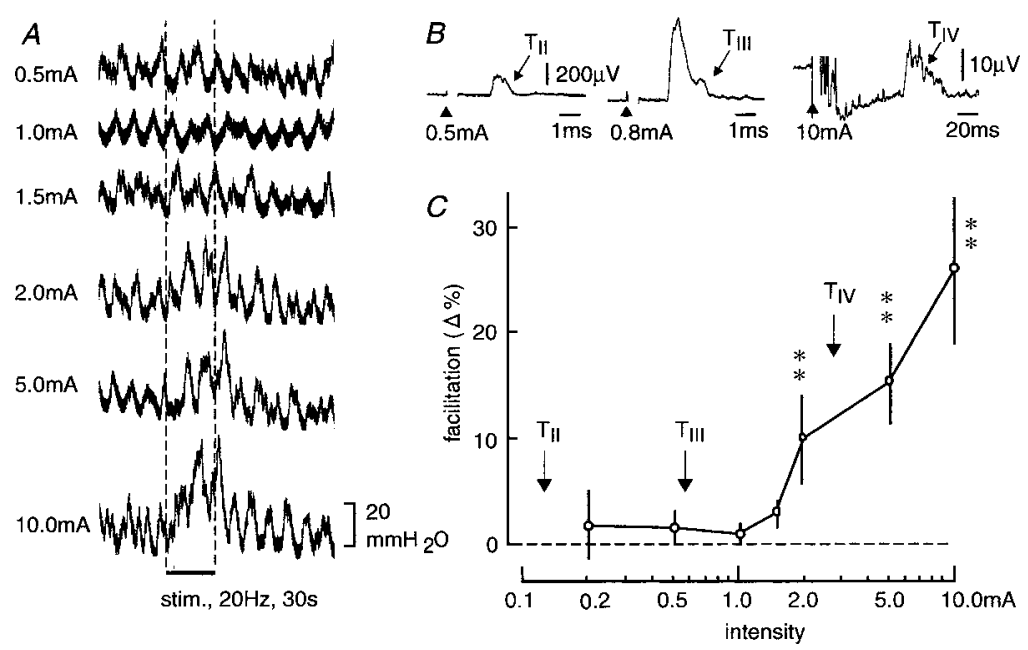

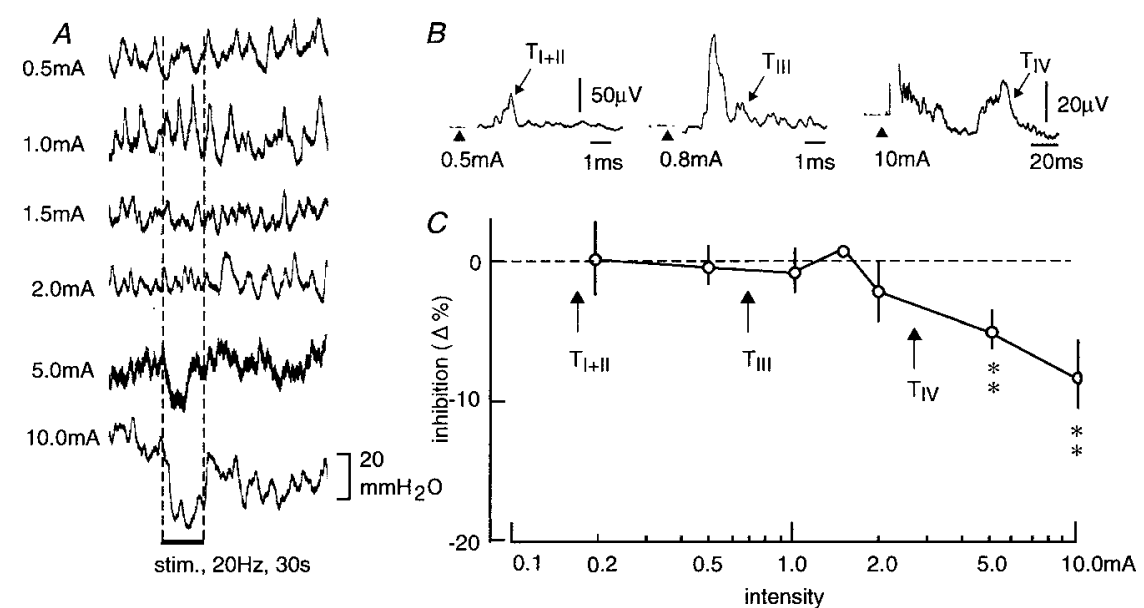

Fig. 2. Effect of EAS of the abdomen at various intensities at $20 \mathrm{~Hz}$ for $30 \mathrm{~s}$ on duodenal motility. B: Sample recordings of the evoked potentials of myelinated and unmyelinated fibers from the T12 intercostal nerve by EAS of the abdomen. $T_{1+11}, T_{|I|}$, and $T_{\mathrm{IV}}$ show the mean threshold intensity of I+II, III, and IV fibers of T11-12 intercostal nerves, respectively. Other details are the same as Fig. 1.
$2.0 \mathrm{~mA}$ produced an increase in duodenal motility. This response was further enhanced by increasing the intensity to $5.0 \mathrm{~mA}$ or more. The mean duodenal pressure (the locus of points midway between adjacent extremes of pressure waves) at the point of maximum deviation during stimulation for each trial was considered to be the response magnitude for that trial and was expressed as a percentage of the prestimulation control value. Changes in the maximum increase of duodenal motility in response to EAS at 2.0, 5.0, and $10.0 \mathrm{~mA}$ were $10.0 \pm 4.3,15.1 \pm 3.8$, and $25.8 \pm 6.9 \%$ (mean \pm SEM), respectively.

The facilitatory responses of duodenal motility evoked by EAS to a hindpaw were abolished after severance of the sciatic and femoral nerves ipsilateral to the hindpaw stimulated.

Compound action potentials recorded from the saphenous and tibial nerves. A single electrical shock to a hindpaw via the acupuncture needles evoked compound action potentials in the saphenous and tibial nerves innervating the ipsilateral hindpaw. The evoked compound potentials, recorded from the peripheral cut end of the saphenous and tibial nerves, were composed of the three main components that conducted along the groups of (1) myelinated I or II or both I and II, (2) myelinated III, and (3) unmyelinated IV nerve fibers (Fig. 1B). The mean thresholds of the action potentials of groups II, III, and IV fibers in the saphenous nerves obtained from the four rats were $0.14(0.12-0.16), 0.55(0.50-0.60)$, and 2.88 (2.00-4.00) mA, respectively. The mean thresholds of the action potentials of groups I and II, group III, and group IV fibers in the tibial nerve obtained from the four rats were $0.15(0.10-0.20), 0.45(0.40-0.50)$, and $2.75(2.50-3.00) \mathrm{mA}$, respectively.

\section{EAS of the abdomen.}

Effects of EAS of the abdomen on duodenal motility. Figure 2A shows typical responses of duodenal motil- ity following EAS at $20 \mathrm{~Hz}$ with various stimulation intensities for $30 \mathrm{~s}$, and Fig. 2C summarizes the responses obtained from all eight rats tested. When the stimulus was less than $2.0 \mathrm{~mA}$, there was no significant response of duodenal motility. EAS at $5.0 \mathrm{~mA}$ produced a decrease in duodenal motility. This response was further augmented when the intensity was increased to $10.0 \mathrm{~mA}$. Changes in the mean maximum decrease of duodenal motility in response to EAS at 5.0 and $10.0 \mathrm{~mA}$ were $6.0 \pm 1.3$ and $9.1 \pm 2.5 \%$, respectively.

The inhibitory response of duodenal motility evoked by EAS to the abdomen was abolished after severing the spinal nerves between the T8 and T13 levels ipsilateral to the stimulated site.

Compound action potentials recorded from the T11-12 intercostal nerves. Compound action potentials evoked by a single electrical shock to the abdomen via the acupuncture needles were recorded from the T11-12 intercostal nerves innervating the ipsilateral abdomen. The evoked compound potentials, recorded from the peripheral cut end of the intercostal nerves in four rats, were composed of the three main components that conducted along the groups of (1) myelinated I and II, (2) myelinated III, and (3) unmyelinated IV nerve fibers (Fig. 2B). The mean thresholds of the action potentials of groups II, III, and IV fibers in the T11-12 intercostal nerves obtained from the four rats were $0.19(0.10-0.25), 0.70$ $(0.50-0.80)$, and $2.75(2.00-3.00) \mathrm{mA}$, respectively.

Effects of EAS on the large, slow, irregular contractions. The effects of EAS on the large, slow, irregular contractions were examined in two rats. Although the large and slow contractions were infrequent and inconsistent, abdominal stimulation inhibited contraction, while hindpaw stimulation induced it. 
Fig. 3. Effect of severance of the autonomic nerves and transection of the spinal cord on excitatory duodenal response of a hindpaw to EAS. A Responses in rats whose spinal cord and autonomic nerves were intact. B: Responses in rats whose vagi were bilaterally cut but whose splanchnic nerves and spinal cord were intact. $\mathbf{C}$ : Responses in rats whose splanchnic nerves were bilaterally cut but whose vagi and spinal cord were intact. D: Responses in rats whose spinal cord was transected but whose autonomic nerves were intact. A1, B1, C1, and D1 show examples of duodenal motility. A2, B2, C2, and D2 show changes in the mean duodenal pressure during stimulation (5.0 and $10.0 \mathrm{~mA}$ ) as the percentage of mean duodenal pressure. The vertical lines drawn in each column represent the SEM. Other details are the same as Fig. 1.
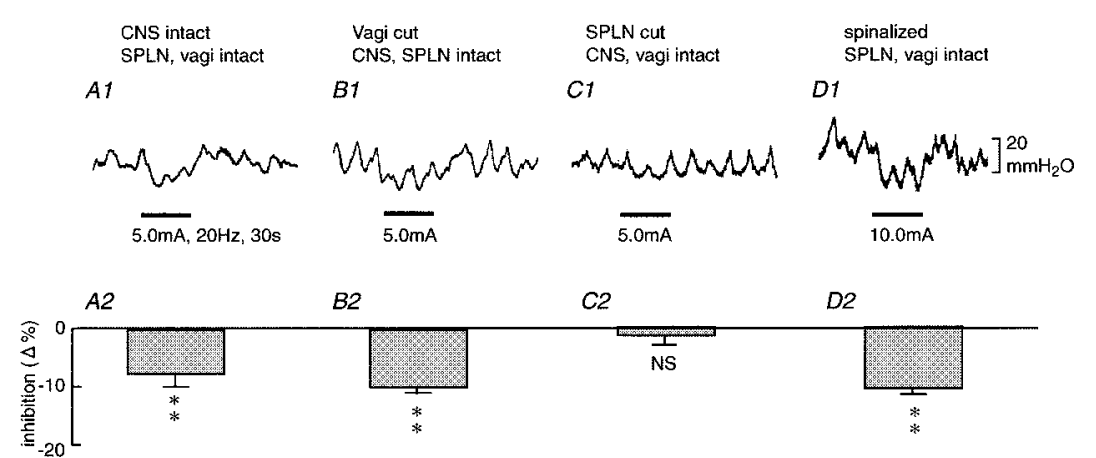

Fig. 4. Effect of severance of the autonomic nerves and transection of the spinal cord on the inhibitory duodenal response of the abdomen to EAS. Other details are the same as Fig. 3.

\section{Effects of severance of autonomic nerves and transection of the spinal cord on duode- nal motility}

Facilitatory duodenal response. The excitatory duodenal response of the hindpaw to EAS at 5.0 and $10.0 \mathrm{~mA}$ was completely abolished after severing the bilateral vagi in all six rats examined (Fig. 3A1, A2, B1, B2). Severing the splanchnic nerve branches to the duodenum did not affect the excitatory duodenal response evoked by hindpaw stimulation in the four cases examined (Fig. 3C1, C2). After the spinal cord was transected at the T2 level, the excitatory duodenal response of the hindpaw to EAS disappeared (Fig. 3D1, D2) in four cases tested.

Inhibitory duodenal response. Severing the bilateral vagi did not affect the inhibitory duodenal response evoked by EAS of the abdomen at 5.0 and $10.0 \mathrm{~mA}$ in five cases examined (Fig. 4A1, A2, B1, B2). Severing the sympathetic nerve branches to the duodenum completely abolished the inhibitory duodenal response evoked by EAS of the abdomen in all four cases examined (Fig. 4C1, C2). After the spinal cord was transected at the T2 level, the inhibitory duodenal response observed during EAS of the abdomen was present in the four cases tested (Fig. 4D1, D2).

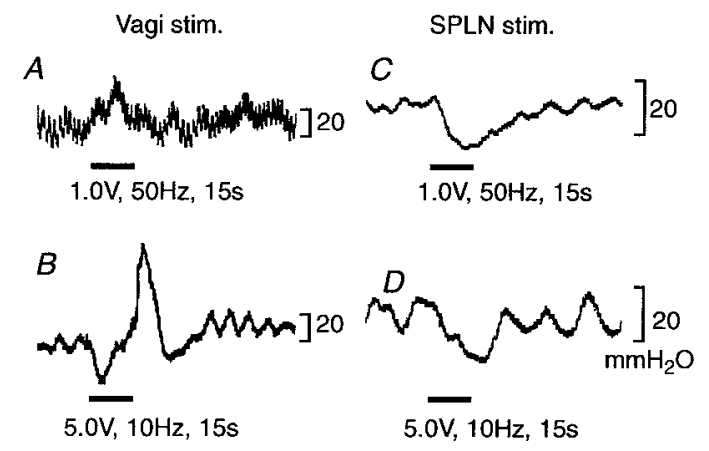

Fig. 5. Effect of electrical stimulation of the vagal and splanchnic nerves. A, B: Sample recordings of duodenal motility in response to vagal nerve stimulation at $1.0 \mathrm{~V}$, $50 \mathrm{~Hz}(\mathbf{A})$ and $5.0 \mathrm{~V}, 10 \mathrm{~Hz}$ (B). C, D: Sample recordings of duodenal motility in response to splanchnic nerve stimulation at $1.0 \mathrm{~V}, 50 \mathrm{~Hz}(\mathbf{C})$ and $5.0 \mathrm{~V}, 10 \mathrm{~Hz}(\mathbf{D})$. The thick horizontal bars on the abscissa indicate the time of stimulation.

\section{Electrical stimulation of autonomic nerves to the duodenum}

Figure 5A and B show typical duodenal motility responses to electrical stimulation of the peripheral cut end of the left cervical vagus for $15 \mathrm{~s}$. Vagus stimulation at $1.0 \mathrm{~V}, 50 \mathrm{~Hz}$ produced an increase in duodenal motility (Fig. 5A), while vagus stimulation at $5.0 \mathrm{~V}$, $10 \mathrm{~Hz}$ produced an early transient decrease in duode- 
nal motility followed by a large increase (Fig. 5B). Figure $5 \mathrm{C}$ and $\mathrm{D}$ show duodenal motility responses to electrical stimulation of the splanchnic nerve for $15 \mathrm{~s}$. Splanchnic nerve stimulation at $1.0 \mathrm{~V}, 50 \mathrm{~Hz}$ and $5.0 \mathrm{~V}$, $10 \mathrm{~Hz}$ produced a decrease in duodenal motility (Fig. $5 \mathrm{C}, \mathrm{D})$.

\section{DISCUSSION}

Many acupoints used for clinical treatment of gastrointestinal disorders are located on the lower legs or in the abdominal area [1, 2, 11-13].

The present study showed that EAS to a hindpaw or abdominal area produced responses of duodenal motility in anesthetized rats after eliminating emotional responses due to EAS. The responses were dependent on the areas stimulated by electro-acupuncture. EAS applied to the abdominal area inhibited duodenal motility, while EAS applied to a hindpaw enhanced it.

The facilitatory responses of duodenal motility following EAS to a hindpaw required intact preparations of vagal nerves, somatic afferent nerves of a hindpaw stimulated by electro-acupuncture, and the spinal cord, but did not require intact preparation of the splanchnic nerve. This evidence indicates that this facilitatory response is a reflex response. The afferents are somatic afferents, the efferents are vagal parasympathetic efferent fibers, and the central structure includes the supraspinal structure.

Koizumi et al. reported that pinching a hindpaw induced an excitatory reflex of jejunal motility via inactivation of intestinal sympathetic nerves in anesthetized rats [14]. It is interesting to note that the efferent pathway of the excitatory duodenal reflex is different from that of the excitatory jejunal reflex.

Repetitive electrical stimulation of efferent vagal fibers with $5.0 \mathrm{~V}$ for $15 \mathrm{~s}$ produced a small decrease in duodenal motility followed by a large increase. Similar findings of biphasic responses when stimulating $\mathrm{B}+\mathrm{C}$ fiber groups of vagal nerves were also reported for gastric motility in rats [15]. This facilitatory component of duodenal motility by vagal stimulation appears to summate and override the inhibitory components in response to EAS to a hindpaw.

The inhibitory responses of duodenal motility following EAS of the abdominal area required intact preparations of splanchnic nerves and somatic afferent nerves of spinal nerves between the T8 and T13 segments stimulated by electro-acupuncture, but did not require intact preparations of bilateral vagal nerves and the spinal cord. This response existed after spinal transection at the T2 segment. Repetitive electrical stimulation of splanchnic nerves for $15 \mathrm{~s}$ produced a monophasic decrease in duodenal motility. Similar findings of a monophasic response when stimulating the B fiber group of splanchnic nerves were reported for gastric motility [15]. This inhibitory component of duodenal motility by splanchnic nerve stimulation appears to summate in response to EAS to the abdominal area.

EAS to a hindlimb in rats has been known to excite various afferent fibers such as groups II [16] and III [17], groups III and IV [9] or groups II, III, and IV [10] afferent fibers. The present experiments demonstrate that stimulating group III and IV afferent fibers, particularly group IV afferent fibers, in saphenous and tibial nerves using EAS to a hindpaw produces an excitatory duodenal reflex response. On the other hand, stimulation of only group IV abdominal nerve using EAS to the abdominal area produces an inhibitory duodenal reflex response.

In conclusion, the present results of anesthetized rats show that applying EAS to a hindlimb and exciting groups III and/or IV afferents produces a supraspinal reflex excitation of duodenal motility via activation of parasympathetic efferents, and that applying EAS to the abdominal area and exciting group IV afferents produces spinal reflex inhibition via activation of sympathetic efferents.

Clinical validity of acupuncture to improve gastrointestinal dysfunction or disorder in humans appears to be attributed to reflex responses of duodenal motility as shown in the present study, and reflex responses of gastric motility [6] and gastric acid secretion [7] in anesthetized animals. In humans, the somatically induced reflex responses of gastrointestinal functions seem to be modulated by emotionally induced responses due to acupuncture stimulation. However, overall gastrointestinal responses of reflex nature and emotional nature following acupuncture appear to be controlled by selecting the location of acupoints and stimulation intensity.

We are grateful to Dr. Yuko Sato, professor of the University of Human Arts and Sciences, for her encouragement in finishing this study.

\section{REFERENCES}

1. O'Connor $J$ and Bensky D: A summary of research concerning the effects of acupuncture. Am J Chin Med 3: 377-394, 1975

2. Li Y, Tougas G, Chiverton SG, and Hunt RH: The effect of acupuncture on gastrointestinal function and disorders. Am J Gastroenterol 87: 1372-1381, 1992

3. Sato A, Sato $Y$, Shimada F, and Torigata $Y$ : Changes in gastric motility produced by nociceptive stimulation of 
the skin in rats. Brain Res 87: 151-159, 1975

4. Kametani $H$, Sato A, Sato $Y$, and Simpson A: Neural mechanisms of reflex facilitation and inhibition of gastric motility to stimulation of various skin areas in rats. J Physiol (Lond) 294: 407-418, 1979

5. Sato $A$, Sato $Y$, and Schmidt RF: The impact of somatosensory input on autonomic functions. Rev Physiol Biochem Pharmacol 130: 166-189, 1997

6. Sato A, Sato Y, Suzuki A, and Uchida S: Neural mechanisms of the reflex inhibition and excitation of gastric motility elicited by acupuncture-like stimulation in anesthetized rats. Neurosci Res 18: 53-62, 1993

7. Noguchi $\mathrm{E}$ and Hayashi $\mathrm{H}$ : Increases in gastric acidity in response to electroacupuncture stimulation of the hindlimb of anesthetized rats. Jpn J Physiol 46: 53-58, 1996

8. Sato $\mathrm{Y}$ and Terui $\mathrm{N}$ : Changes in duodenal motility produced by noxious mechanical stimulation of the skin in rats. Neurosci Lett 2: 189-193, 1976

9. Noguchi E, Ohsawa H, Kobayashi S, Shimura M, Uchida S, and Sato Y: The effect of electro-acupuncture stimulation on the muscle blood flow of the hindlimb in anesthetized rats. J Auton Nerv Syst 75: 78-86, 1999
10. Ohsawa $H$, Yamaguchi $S$, Ishimaru $H$, Shimura $M$, and Sato Y: Neural mechanism of pupillary dilation elicited by electro-acupuncture stimulation in anesthetized rats. J Auton Nerv Syst 64: 101-106, 1997

11. Sodipo JOA and Falaiye JM: Acupuncture and gastric acid studies. Am J Chin Med 4: 356-361, 1979

12. Mann F: Textbook of Acupuncture, William Heinemann Medical Books, London, 640 pp, 1987

13. Stux $G$ and Pomeranz B: Basics of Acupuncture, Springer-Verlag, Berlin, 322 pp, 1998

14. Koizumi K, Sato A, and Terui N: Role of somatic afferents in autonomic system control of the intestinal motility. Brain Res 182: 85-97, 1980

15. Aihara $Y$, Nakamura $H$, Sato $A$, and Simpson A: Relations between various fiber groups of vagal and splanchnic nerves and gastric motility in rats. Neurosci Lett 10: 281-286, 1978

16. Toda K: Effects of electro-acupuncture on rat jaw opening reflex elicited by tooth pulp stimulation. Jpn J Physiol 28: 485-497, 1978

17. Kawakita $\mathrm{K}$ and Funakoshi M: Suppression of the jawopening reflex by conditioning A-delta fiber stimulation and electroacupuncture in the rat. Exp Neurol 78: 461-465, 1982 